\title{
164 FREQUENCY OF BLADDER, BOWEL AND SEXUAL DYSFUNCTION IN NMO
}

Kerry Mutch, Abigail Methley, Steven Zhao, Liene Elsone, Anton Emmanuel, Jalesh Panicker, Anu Jacob. The Walton Centre NHS Foundation Trust

10.1136/jnnp-2014-309236.164

Background The impairment of mobility due the severe myelitis in NMO is well known, but the effect on bladder, bowel and sexual function is not reported. We report the frequency of these problems in NMO.

Method 50 patients [42 females) with aquaporin-4 antibody positive NMO spectrum disorder, all with myelitis, were studied. They completed validated organ-specific questionnaires: Bladder Control Scale (BLCS), Bowel Control Scale (BWCS), Sexual Satisfaction Scale (SSS).

Results Bladder symptoms were reported in $78 \%$ and $44 \%$ experienced severely restricted lifestyle due to these. Similarly $80 \%$ had high bowel scores (54\% constipation) and $24 \%$ experienced lifestyle impairment. Of the 29 patients in a relationship $12(41 \%)$ were dissatisfied with their sexual life, four of five men had erectile dysfunction.

Conclusion This first ever report on the extent of pelvic organ dysfunction in NMO identified significant and oft hidden morbidity. The importance of assessment of these symptoms is emphasised by the availability of treatments for them. It also highlights the importance of treating and preventing relapses in NMO. 